\title{
La metodología de LEGO Juego Serio como herramienta pedagógica en escenarios educativos, empresariales y sociales.
}

\author{
Linda Bustos Parra ${ }^{26}$ \\ Manuel Méndez Pinzón' 27
}

\section{Necesidad de formación}

$\mathrm{L}$ a búsqueda de nuevos métodos de formación en los escenarios académicos empresariales y sociales a partir del uso de metodologías que involucren el desarrollo de habilidades cognitivas, estratégicas, desarrollo de la creatividad, el aprendizaje colaborativo y el trabajo en red, hace necesario el uso de herramientas pedagógicas que permita la construcción de un aprendizaje significativo, en donde los participantes sean parte activa de este proceso; es por ello que la aplicación de metodologías como LEGO Juego Serio permiten que el participante sea el eje central de este aprendizaje, cambiando el paradigma del profesor como eje central del proceso de aprendizaje.

De acuerdo con los planteamientos dados anteriormente, surge la siguiente pregunta de investigación: ¿cómo la implementación de la metodología LEGO Juego Serio desarrolla habilidades cognitivas y estratégicas en escenarios educativos, empresariales y sociales?

El presente ejercicio de investigación se propone analizar cómo la metodología LEGO Juego Serio desarrolla habilidades cognitivas y estratégicas en escenarios educativos, empresariales y sociales. Para ello se centrará en identificar teorías que fundamentan el uso de esta metodología para desarrollo de habilidades cognitivas y estratégicas en escenarios educativos, empresariales y sociales; en diseñar y aplicar los instrumentos de investigación en los mismos escenarios; y, por último, en interpretar y analizar los datos arrojados en la aplicación de los instrumentos.

26. Docente Asociada Facultad Ciencias Humanas y Sociales. Magistra en Educación Pontificia Universidad Javeriana.linda.bustos@unimilitar.edu.co

27. Profesor Tiempo Completo, Facultad Negocios Gestión y Sostenibilidad. Magister en Administración, MBA. Correo electrónico: mmendez@poligran.edu.co 


\section{Marco teórico}

En el presente capítulo se tratarán los diferentes conceptos en los que se basa este trabajo de investigación; en la primera parte se presenta las diferentes categorías relacionadas con el aprendizaje, los procesos cognoscitivos básicos, la definición de la metodología LEGO Juego Serio, terminando con el proceso y desarrollo de la metodología.

\section{Categorías relacionadas con el aprendizaje}

Procesos cognitivos básicos: se refiere al proceso involucrado en el procesamiento de información, como atención, percepción, codificación, almacenaje y recuperación (Hernandez y Barriga, 1999).

Los procesos cognitivos básicos son indispensables para la ejecución de todos los otros procesos de orden superior. Aquellos se ven poco afectados por el desarrollo; desde edad muy temprana, los procesos y funciones cognitivos básicos parecen estar presentes en su forma definitiva, cambiando relativamente poco con el paso de los años. Una excepción que destaca es la referida a la supuesta capacidad creciente de la memoria de trabajo (operador M: espacio mental), con la edad (de la niñez temprana a la adolescencia), tal como lo han demostrado algunos investigadores neo piagetianos, por ejemplo R. Case -y -J-Pascual=Leone.

- Base de conocimientos: son los conocimientos previos que poseen los individuos; resaltando experiencias, conceptos y principios que son organizados jerárquicamente; lo anterior también es conocido como saber por Brown (1975), y según Ausubel (1968), en su teoría de aprendizaje significativo, como conocimientos previos.

- Conocimiento estratégico: tiene que ver con las estrategias de aprendizaje. Brown (1975), lo define como: "aprender cómo conocer".

En primer lugar, puede decirse también que existen formas más generales y otras más específicas; estas estrategias son aplicables a varios dominios de aprendizaje, mientras que otras tienden a restringirse a contenidos muy particulares. Esto ha llevado a algunos autores a clasificar entre estrategias generales y específicas, aunque en muchas ocasiones se ha vinculado a las estrategias generales con las llamadas metacognitivas (véase, por ejemplo, Kirby, 1984, citado por Nisbet y Schucksmith, 1986, quien utiliza el término "microestrategias", para las estrategias cognitivas o de aprendizaje, y "microestrategias", para el caso de estrategias metacognitivas). 
- Conocimiento metacognitivo: es el conocimiento que se posee sobre los propios procesos cognitivos, cuando se aprende, se recuerda o se soluciona algún problema. Brown (1975), lo describe como: "conocimiento sobre el conocimiento".

- Reconstrucción del conocimiento: (Aprendizaje Significativo): se basa en la teoría de aprendizaje significativo de David Ausubel, en la cual se tienen en cuenta los conocimientos previos de los educandos y la reconstrucción que se lleva a cabo con la unión del nuevo aprendizaje.

Teniendo en cuenta el objetivo principal de la teoría del aprendizaje significativo, el que fundamenta la importancia que tienen las experiencias y conocimientos previos de los estudiantes para que, por medio de la guía del docente, se creen ambientes que generen nuevos aprendizajes o la reconstrucción del mismo; se ha evidenciado que muchas metodologías han fracasado en el proceso de enseñanza aprendizaje, ya que se tiene desconocimiento o no se les da la importancia que tienen los procesos cognitivos, afectivos y meta cognitivos a los estudiantes, lo cual juega un papel fundamental como conocimientos previos, puesto que el proceso cognitivo es un conjunto de operaciones mentales que ayudan a la adaptación y al mantenimiento en el mundo que nos rodea; de estos procesos surgen dos clases:

- Procesos cognitivos básicos: son la base del procesamiento de la información, permite que se adapte para posterior trabajo con ella; entre estos procesos están la atención, la percepción y la memoria.

- Procesos cognitivos superiores: estos requieren del máximo nivel de integración de la información, se apoya en los procesos básicos como insumo para su funcionamiento; entre estos tipos de pensamiento se encuentran la memoria, el lenguaje, la creatividad, el aprendizaje y motivación.

Así mismo, las emociones y la dimensión afectiva no pueden ser desligadas del aprendizaje, puesto que esto hace parte del conocimiento previo que se ha adquirido por medio de vivencias y experiencias previas.

Y la metacognición, definida como el conocimiento del cómo aprendemos es fundamental en el desarrollo del aprendizaje significativo, ya que el estudiante emplea una manera intencional para aprender y solucionar problemas, creando sus propias estrategias de aprendizaje.

\section{Metodología LEGO Juego Serio}

La metodología LEGO Juego Serio surge como solución a problemas de creatividad evidenciados en las juntas de planeación estratégica de la compañía Lego; debido a esto, el dueño decide empezar a implementar el uso de las fichas en las juntas, obteniendo excelentes resultados. 
Esta metodología se ha implementado en diferentes compañías y en múltiples escenarios, obteniendo resultados como fomento del trabajo en equipo, aumento de la creatividad y la innovación, generación de compromiso con los procesos de la empresa y el cumplimiento de objetivos, etc.

La metodología de LEGO Juego Serio fomenta la solución de problemas de manera creativa; desarrollando algunas actividades que permiten:

- Tener conocimiento del problema.

- Dar posibles soluciones a través de la construcción de LEGO.

- Evaluar las posibles soluciones.

\section{Establecer relación con otras soluciones.}

\section{Desarrollo de la metodología}

LEGO Juego Serio se lleva a cabo por medio de un facilitador, quien guía el proceso motivando a los participantes a: a) plantear un desafío o pregunta, b) responder construyendo un modelo, c) compartir las construcciones y diseñar estrategias, y d) finalmente, fomentar la reflexión conectando las creaciones con la realidad.

A continuación, se mencionarán algunos pasos que se deben tener en cuenta para la aplicación de la metodología como herramienta para la creación de valor compartido:

1. Etapa de observación: los participantes deben tener claridad en cuanto al problema que quieren trabajar.

2. Etapa de descripción: se deben formar grupos de acuerdo con el total de personas que se encuentren en la sesión, y por medio de la utilización de las fichas de LEGO, los participantes deben hacer una construcción acerca del problema identificado en la etapa anterior. Después, se debe crear una historia que permita describir el significado de dicha construcción.

3. Etapa de ideación: los participantes, por medio de utilización de las fichas LEGO, deben proponer posibles soluciones al problema, y mediante una historia narrar el significado de la construcción.

4. Etapa de sustracción: después de describir los componentes y características más importantes del problema, los participantes deben aplicar la sustracción, en la que restan algún paso o característica del problema con el fin de obtener mejores resultados.

5. FEtapa de división: en esta etapa se deben dividir componentes del problema con el fin de dar soluciones innovadoras al mismo. Posteriormente, se debe socializar por medio de una historia. 
6. Etapa de unificación: se deben unificar características o componentes del problema con el fin de innovar y dar solución al problema.

7. Solución del problema: finalmente, todos los grupos unen sus construcciones con el fin de relacionarlas y formar una solución más completa del problema. Esta se debe socializar por medio de una historia, explicando el significado de la construcción.

La metodología LEGO Juego Serio se ha implementado para la solución de problemas en diferentes escenarios; entre estos: académicos, empresariales y sociales, ya que se apoya en la teoría del construccionismo y constructivismo para fomentar la creatividad promoviendo el uso de las dos manos, por ende, los dos hemisferios del cerebro se conectan y se dan procesos mucho más creativos, contando con la parte imaginativa del hemisferio derecho y la parte razonable del hemisferio izquierdo.

En el escenario educativo, según la teoría del constructivismo de Vygotsky, los estudiantes desarrollan aprendizajes y adquieren conocimiento de una manera más significativa cuando, por medio del lenguaje, comparten con sus pares experiencias; lo cual se evidencia todo el tiempo con la implementación de LEGO, ya que los participantes recrean construcciones con las fichas y posteriormente las justifican por medio de la creación de historias o metáforas que dan un significado a la construcción, y de esta manera no solamente reconstruyen el conocimiento partiend o de sus experiencias previas, sino que aprenden nuevos conceptos de las experiencias de otros. Teniendo en cuenta lo anterior (Carrera y Mazzarella, 2001, p.42), afirman que: “Uno de los aportes más significativos de la obra de Vygotsky lo constituye la relación que establece entre el pensamiento y el lenguaje. Señala que en el desarrollo ontogenético ambos provienen de distintas raíces genéticas"

Es por ello que LEGO promueve una estrecha relación entre el pensamiento y el lenguaje, lo cual genera un desarrollo cognitivo y la adquisición de nuevos aprendizajes. Cuando se es capaz de explicar algo por medio del lenguaje es porque ya se ha comprendido, se ha apropiado, y de esta manera con palabras propias se puede explicar; esto ocurre cuando se cuentan las historias acerca de las construcciones realizadas.

Por otro lado, en un escenario empresarial, la metodología LEGO Juego Serio cumple un papel muy importante en el desarrollo de la planeación estratégica e identificación de oportunidades de creación de estrategias, ya que su implementación logra:

- Promover la participación de todos los miembros de un equipo: gracias a que LEGO se identifica como un juego, las personas se muestran más seguras, lo cual genera mayor participación.

- Promueve el respeto por las ideas de otros: la actividad de jugar ubica a todos los participantes como iguales, sin importar cargos ni estatus dentro del juego, lo cual hace que todas las ideas tengan el mismo valor e importancia.

- Aumenta la creatividad y la innovación: el hecho de pensar al mismo tiempo que actuar con las manos mejora la capacidad de desarrollar ideas y ser más creativo. 
- Mayor compromiso con el cumplimiento de objetivos: el hecho de fomentar la participación de todos los miembros del equipo promueve mayor sentido de pertenencia y orientación al cumplimiento de objetivos comunes.

Por otra parte, en el ámbito social da grandes aportes en el trabajo colaborativo y en la interacción social; fomenta procesos empáticos y fortalece el proceso de comunicación, haciendo que sea mucho más asertiva, lo que facilita la detección de oportunidades, así como el desarrollo de soluciones con un mayor impacto e innovación.

Con las construcciones de LEGO, los participantes logran tener procesos de autoconocimiento, dando características a los personajes que se crean en las construcciones; por otro lado, clarifica intenciones, deseos, gustos y emociones frente a situaciones diversas creadas en las historias.

Adicionalmente, LEGO Juego Serio fortalece las relaciones interpersonales, fomentando procesos de empatía por medio de la validación emocional que ocurre en el momento en el que los participantes logran ponerse en el lugar de los personajes de las construcciones; de esta manera, promueven el ejercicio de ponerse en el lugar del otro en su cotidianidad.

De acuerdo con lo anterior, la implementación de la metodología LEGO Juego Serio fortalece los procesos de comunicación, ya que hacer el ejercicio de crear las historias y de explicar a los demás el significado de las construcciones desarrolla competencias comunicativas, las cuales se van evidenciar en procesos de comunicación más asertiva, en donde el emisor emite mensajes con claridad y logra que el receptor entienda claramente el mensaje, evitando malas interpretaciones y futuros malos entendidos.

De igual modo, se concluye que la implementación de la metodología permite identificar problemas en todos los escenarios nombrados anteriormente; esta ha sido una herramienta que se ha probado en el aula de clase con estudiantes de pregrado, mostrando excelentes resultados no solo en el proceso de aprendizaje, sino en el fortalecimiento de habilidades sociales y comunicativas, ya que permite ponerse en el lugar del otro y fomentar el trabajo colaborativo y de equipo.

Finalmente, LEGO se ha implementado en las empresas para trabajar liderazgo, fortaleciendo los equipos de trabajo y proyectando los logros de la empresa como fin común, haciendo más productivo el trabajo y obteniendo mejores resultados.

\section{Metodología}

Se realizará en varias fases, empezando por la investigación preliminar y rastreo bibliográfico, pasando por una fase de campo donde se realizará un levantamiento y análisis de información cualitativa; por último, se procesará la información levantada para su correspondiente análisis. 
La metodología que se ha seguido para esta investigación se agrupa en distintas etapas, empezando por una exploración metodológica para la elaboración del modelo metodológico y contextual, seguido por el levantamiento de los instrumentos, luego por una fase de campo en la cual se realizará un levantamiento de información primaria y secundaria. Al final, procesamiento y análisis de la información levantada.

El presente estudio corresponde a un diseño cualitativo de tipo descriptivo. De acuerdo con Sampieri (2016), los estudios descriptivos buscan especificar características importantes de personas, grupos y comunidades que son sometidos a un análisis; teniendo en cuenta este caso particular, se busca describir los comportamientos de la muestra después de aplicar la metodología LEGO.

\section{Muestra}

Según Sampieri (2014), se definió como una muestra no probabilística, o muestra dirigida, que se supone un procedimiento de selección informal y es utilizada en diversas investigaciones de tipo cualitativas, donde se selecciona participantes por uno o varios propósitos.

En este estudio participaron 56 estudiantes, entre los 16 y 21 años, de los programas de Ingeniería civil, Administración de Empresas, Negocios internacionales y Técnico en gestión de aerolíneas de las instituciones Universidad Militar Nueva Granada y el Politécnico Grancolombiano.

En la tabla a continuación se evidencia la distribución de la muestra de la población que participó en el presente estudio: características de edad, género y por procedencia de la institución educativa.

Tabla 4. Características de la población estudiada

\begin{tabular}{|l|c|c|c|}
\hline \multicolumn{1}{|c|}{ Procedencia } & \multirow{2}{*}{ Edad } & \multicolumn{2}{c|}{ Género } \\
\cline { 3 - 4 } & & Femenino & Masculino \\
\hline $\begin{array}{l}\text { Institución A } \\
\text { Universidad Militar Nueva Granada }\end{array}$ & $16-21$ & 17 & 13 \\
\hline $\begin{array}{l}\text { Institución B } \\
\text { Politécnico Grancolombiano }\end{array}$ & $18-22$ & 12 & 14 \\
\hline Total & & 29 & 27 \\
\hline
\end{tabular}

Fuente: elaboración propia (2018). 


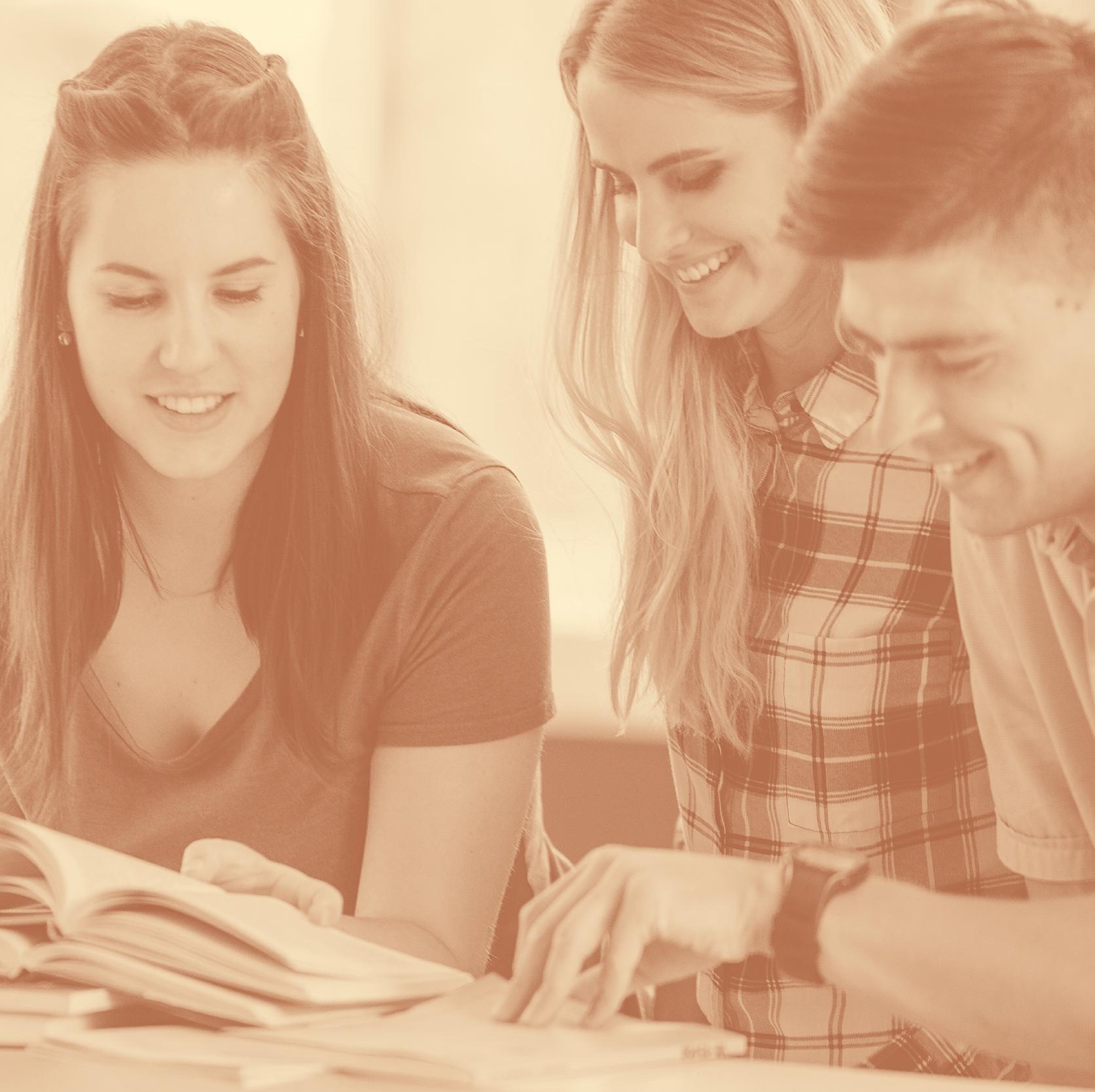




\section{Variables y categorías de análisis}

Partiendo del marco teórico que fundamenta este estudio, se identificaron las siguientes categorías de análisis para determinar cómo la implementación de la metodología LEGO desarrolla habilidades cognitivas y estratégicas en escenarios educativos y empresariales:
a. Procesos cognitivos básicos.
b. Base de conocimientos.
c. Conocimiento estratégico.
d. Conocimiento metacognitivo.
e. Reconstrucción del conocimiento.
f. Creación de metáforas y de historia.

\section{Instrumentación}

Para el desarrollo de este estudio se utilizaron los "diarios de campo", en los cuales se registró toda la información de forma sistemática, teniendo en cuenta cada una de las categorías de análisis, una vez terminada cada una de las intervenciones.

Según Sampieri (2014, p.374), es común que las anotaciones se registren en lo que se denomina diario de campo o bitácora, que es una especie de diario personal, donde además se incluyen anotaciones de la reactividad de los participantes, cambios inducidos por el investigador, problemas en el campoy situaciones inesperadas, por lo que se debe tener en cuenta:

a. Descripciones iniciales y posteriores del ambiente, que abarcan lugares, personas, relaciones y eventos.

b. Mapas.

c. Diagramas, cuadros y esquemas (secuencias de hechos o cronología de sucesos, vinculaciones entre conceptos del planteamiento, redes de personas, organigramas, etcétera).

d. Listado de objetos o artefactos recogidos en el contexto, así como fotografías y videos que fueron tomados (indicando fecha y hora, y por qué se recolectaron o grabaron y, desde luego, su significado y contribución al planteamiento).

e. Aspectos del desarrollo de la investigación (cómo vamos hasta ahora, qué nos falta, qué debemos hacer).

A continuación, se muestra el instrumento creado usado en el proceso de toma de datos. 
Tabla 5. Diario de campo

\begin{tabular}{|l|l|l|l|}
\hline Categoría de análisis & Temática & Actividad & Observaciones \\
\hline $\begin{array}{l}\text { a. Procesos cognitivos básicos. } \\
\text { b. Base de conocimientos. }\end{array}$ & & \\
\hline c. Conocimiento estratégico. & & \\
\hline $\begin{array}{l}\text { d. Reconstrucción del } \\
\text { conocimiento. }\end{array}$ & & \\
\hline e. Creación de metáforas \\
y de historias.
\end{tabular}

Fuente: elaboración propia a partir del marco teórico (2018).

\section{Resultados}

A continuación se presentarán los principales resultados, análisis y la posterior interpretación del trabajo de campo en las dos instituciones. En primer lugar, se realizó la toma de los datos mediante la metodología descrita anteriormente.

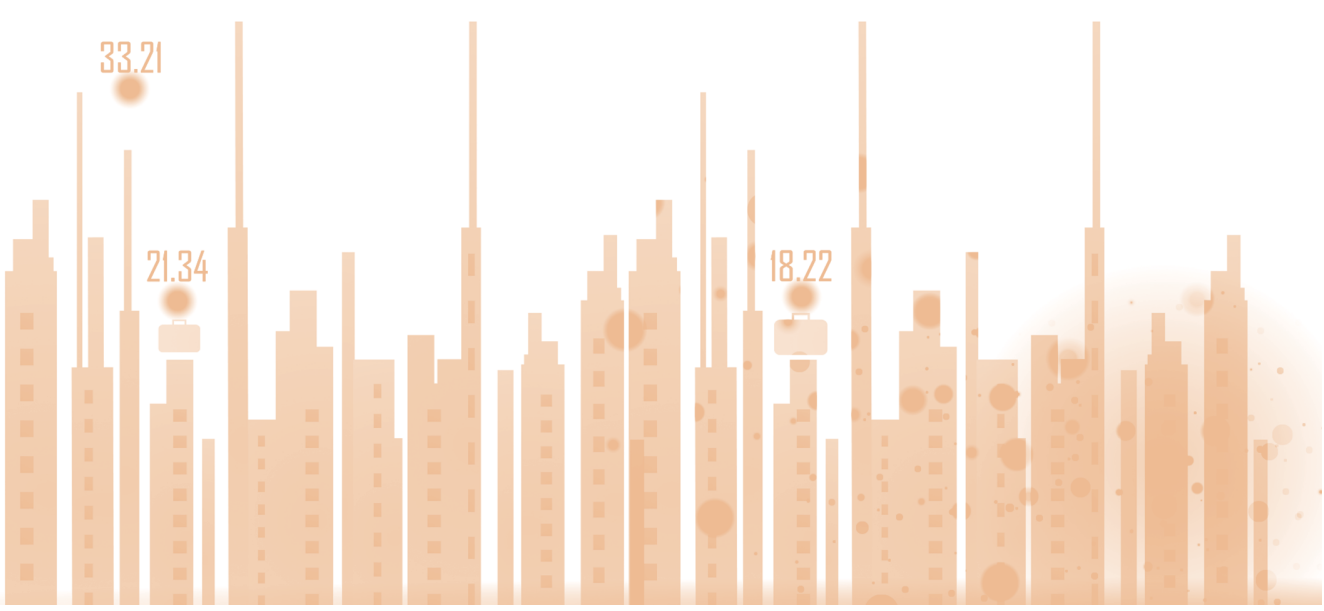




\section{Temática}

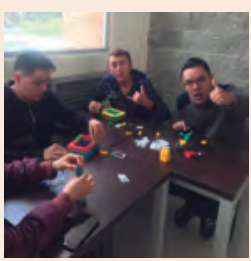

\section{Actividad}

Desarrollo del proyecto de vida personal, incluyendo reconocimiento de la historia de vida y el planteamiento del futuro soñado.

Por otro lado, se realizó una reflexión acerca del aporte social que se daría con el rol profesional de cada uno.

\section{Actividad 1. Observación}

1. Se propuso la descripción de cada persona, por medio de la construcción de una torre de fichas de LEGO.

2. Posteriormente, cada persona debía contar al grupo por qué la torre lo definía y en qué aspectos lo hacía.

\section{Actividad 2. Descripción}

Se planteó la construcción de la situación actual del proyecto de vida particular.

\section{Actividad 3. Ideación}

1. En esta etapa se propone que los estudiantes creen un escenario ideal a futuro, en donde visualicen dicho momento de la mejor manera.

\section{Actividad 4. La sustracción}

1. En este escenario, los estudiantes deben eliminar algún aspecto de la vida de cada uno con el que no estén a gusto.

\section{Actividad 5. La división}

1. Se descompone el escenario ideal de futuro, junto a las posibles estrategias en varias etapas, buscando el análisis desde diferentes perspectivas.

\section{Actividad 6. La unificación}

1. Se elabora la unificación de estrategias para la planeación de futuro, y así llegar a una construcción en consenso, buscando el trabajo colaborativo y en red.

Fuente: elaboración propia a partir del diario de campo Universidad Militar Nueva Granada (2018). 


\section{Categoría} de análisis
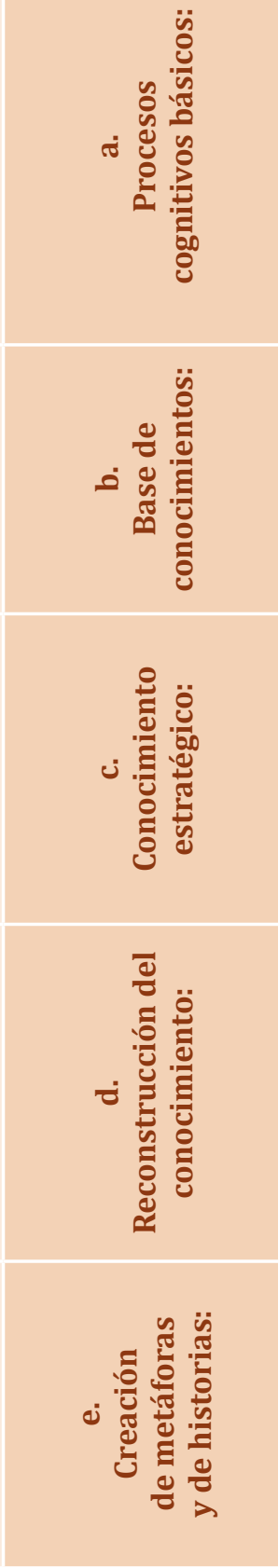

\section{Resultados}

Se evidenció que, mediante la metodología de LEGO Juego Serio, los participantes desarrollaron los procesos cognitivos básicos de la siguiente manera:

En la construcción de todas las actividades, los estudiantes por medio de la percepción- asimilaron información como las formas, tamaños colores, dando un significado a estos que se evidenció en la socialización de las construcciones, en donde explicaban el significado de cada una de ellas.

Por otro lado, se visualizaron procesos de concentración altos en el momento de la construcción.

En las construcciones de la metodología se evidenció que los participantes resaltaron experiencias, sentimientos y conocimientos previos acerca del tema.

En la planeación de futuro de proyecto de vida, los estudiantes diseñaron estrategias a corto, mediano y largo plazo. Por medio de las fichas lograron ser más estratégicos desde todas las dimensiones del ser humano, ampliando mucho más la construcción de futuro.

Por medio de los conocimientos previos que resaltaron los participantes, y con los nuevos adquiridos por medio del docente y los otros participantes, se desarrolló una nueva construcción del mismo, unificando y reestructurando conceptos mucho más elaborados.

A partir del uso de las fichas LEGO fue evidente la creación de metáforas e historias, permitiendo la creatividad en el desarrollo de los escenarios y las diferentes probabilidades de éxito en cada uno de ellos. 


\section{Temática}

Elaboración de estrategias empresariales:

- Diagnóstico empresarial.

- Estrategias empresariales.

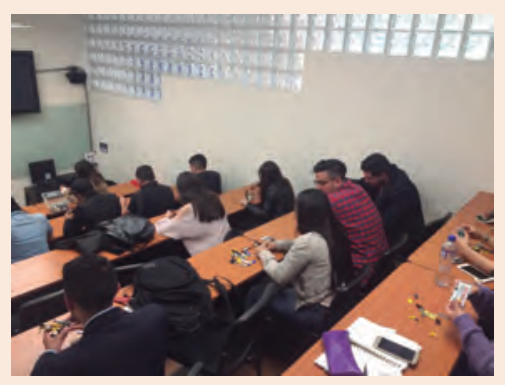

\section{Actividad}

A continuación, se describen las etapas que se presentan en el desarrollo de la metodología LEGO Juego Serio:

\section{Actividad 1. Observación}

Descripción de la situación actual de la empresa.

\section{Actividad 2. Descripción.}

En los grupos de trabajo describen la situación real de la empresa.

\section{Actividad 3. Ideación.}

Se construye la posible solución o escenario ideal.

\section{Actividad 4. Sustracción.}

A partir del escenario ideal, sustraen elementos para el análisis de otras probabilidades.

\section{Actividad 5. División.}

Se descompone el escenario ideal en varios componentes, junto con las posibles estrategias, buscando el análisis desde diferentes perspectivas.

\section{Actividad 6. Unificación.}

Se elabora la unificación de las estrategias de los grupos para llegar a una construcción en consenso, buscando el trabajo colaborativo y en red. 


\section{Categoría de análisis}

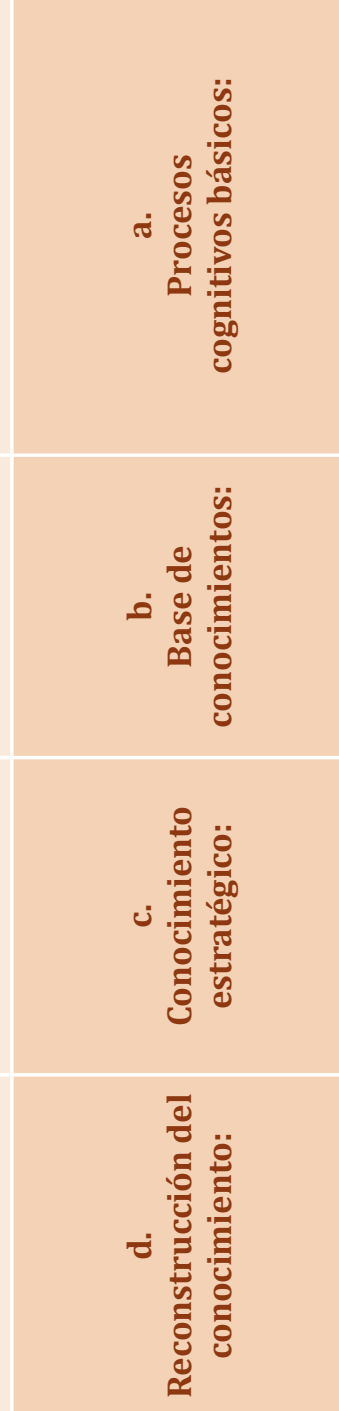

\section{Resultados}

Se evidenció que por medio de la construcción con la metodología LEGO Juego Serio, los participantes desarrollaron los procesos cognitivos de la siguiente manera:

Desarrollo de percepción y visualización, buscando un significado a las figuras, representando la situación real de la empresa objeto de estudio.

También se evidenció un alto nivel de concentración por parte de los participantes en el momento de la construcción.

En la construcción se evidenció un alto conocimiento de la empresa objeto de estudio, así como la fundamentación teórica previa.

Los participantes diseñaron estrategias a corto, mediano y largo plazo, evidenciando su aplicación en las áreas funcionales de la empresa.

Utilizando el conocimiento previo de los participantes, sumado con el conocimiento tácito del docente, se elaboró un escenario ideal para la empresa de estudio, donde se evidenció la creación y trabajo colaborativo en red.

A partir del uso de las fichas LEGO fue evidente la creación de metáforas e historias, permitiendo la creatividad en el desarrollo de los escenarios y las diferentes probabilidades de éxito en cada uno de ellos. 
La implementación de LEGO Juego Serio como una metodología de enseñanza en el aula de pregrado, dejó resultados significativos en los participantes, ya que permitió destacar los conocimientos previos, resaltando emociones, sentimientos y preconceptos, los cuales fueron socializados por medio de historias y comparaciones desarrolladas con las metáforas. Por otro lado, esta metodología motivó la participación de todos los estudiantes y la colaboración del docente, dando igual nivel de importancia a los aportes de cada uno.

Así mismo, se evidencia un aporte para el desarrollo del lenguaje, ya que los participantes usaron palabras más estructuradas en la creación de historias, motivando el enriquecimiento del léxico mediante la socialización e intercambio de historias.

Además, esta metodología promueve el trabajo colaborativo, en el que todos los participantes intervienen con sus comentarios e ideas, desarrollando el pensamiento creativo y motivando el pensamiento divergente, ya que ante un problema se proponen diversas posibles soluciones.

\section{Conclusiones}

La metodología de LEGO Juego Serio en el ámbito educativo da aportes muy importantes en el desarrollo de procesos cognitivos básicos, ya que promueve el desarrollo de la percepción, la memoria y la concentración; igualmente, fortalece los procesos cognitivos superiores, suscitando el pensamiento comprensivo, crítico, creativo y sistémico.

La implementación de LEGO Juego Serio tiene un aporte social en la formación integral, pues fomenta el trabajo colaborativo, fortaleciendo las habilidades blandas, promoviendo la escucha y procesos de empatía y comunicación asertiva.

El desarrollo de esta metodología posibilita crear ambientes de aprendizaje en el aula, en donde prima la motivación y el interés por parte de los participantes; aquí el docente es un mediador y es quien guía dicho proceso de enseñanza- aprendizaje orientando la actividad hacia el cumplimiento de los objetivos propuestos.

\section{Referencias}

Ausubel, D. (1968). Educational Psychology: A Cognitive Approach. NY: Holt.

Brown, A. L. (1980). Metacognitive development and reading. Theoretical issues in reading comprehension: Perspectives from cognitive psychology, linguistics, artificial intelligence, and education, 453-481. 
Carrera, B., y Mazzarella, C. (2001). Vygotsky: enfoque sociocultural. Educere, 5(13).

Díaz, B. F. y Hernández R., G. (1999). Estrategias docentes para un aprendizaje significativo. México: McGraw Hill.

Hernández Sampieri, R., Fernández Collado, C., y Baptista Lucio, P. (2014). Metodología de la investigación. Sexta Edición. México: Mc Graw Hill. México.

Kirby, J.R. (1984). Cognitive strategies and educational performance. Orlando: Academic Press.

Nisbet, J. y Shucksmith, J. (1986). Learning strategies. London: Routledge \& Kegan Paul.

Robertson, D. (2018). Lessons from LEGO: What do you do when your current growth phase ends? Recuperado de: https://theleadershipnetwork.com/article/lessons-from-lego-what-do-you-do-when-your-current-growth-phase-ends

Rosenthal, N. (2015). LEGO's Strategic Blunder: How they Failed to Own Adjacent Markets to Execute their Strategy. Recuperado de: https://www.advantexe.com/blog/a-business-acumen-example-legos-strategic-blunder

Vigotsky, L.S. (1960). El desarrollo de los procesos psicológicos superiores. México: Ed. Juventud.

Vigotsky, L.S. (1973). Pensamiento y lenguaje. Teoría del desarrollo cultural de las funciones psíquicas. Nicaragua: Ed. Pléyade.

Vigotsky, L.S. (1993). Obras escogidas. Tomo II. Madrid: Visor.

Vigotsky L.S. (1995). El problema del desarrollo de las funciones psíquicas superiores. Obras escogidas. Tomo III. Madrid: Visor.

Weverbergh, R. (s.f.). 6 things Lego can teach you about design and innovation. Recuperado de: http://www.whiteboardmag.com/what-lego-can-teach-you-about-design-andinnovation/ 